\title{
Species composition and diversity of aero-terrestrial algae and cyanobacteria of the Boreč Hill ventaroles
}

\author{
Pavel ŠKALOUD \\ Department of Botany, Faculty of Science, Charles University, Benátská 2, CZ-12801, Prague 2, Czech \\ Republic; e-mail: skaloud@natur.cuni.cz;
}

\begin{abstract}
The algal flora of the Boreč Hill ventaroles was examined and compared with the flora of their close surroundings. In comparison to unaffected sites, the ventaroles differ in seasonal temperature fluctuation as well as in soil $\mathrm{pH}$. Winter exhalations caused by continual air circulation in the cranny system of rock massive result in a significant increase in air temperature and soil $\mathrm{pH}$. The ventaroles were inhabited by a markedly richer and more diversified algal flora. Over half of investigated species occurred only in the ventaroles, including all chrysophyte, eustigmatophyte and desmid taxa. In contrast to the unaffected sites, different algal populations were discovered in the ventaroles. The investigated species could be separated into two groups: ventarole-specific ones preferentially occurred in both the ventaroles, and those occurred only in a large ventarole. There, water condensation in moss plants during winter exhalations enables a short-term occurrence of several algal species preferring aquatic environments. The diatom flora of the ventaroles resembles well the species composition found in the caves. By contrast, the desmid flora is rather similar to algal communities found in ephemeral water bodieslike temporary peat bog pools or dripping rocks. Thus, the Boreč Hill ventaroles represent a unique type of biotope, with a specific algal flora adapted to periodical periods of warm, moist air exhalations of several months' duration.
\end{abstract}

Key words: aerophytic algae, Chlorophyta, desmids, diatoms, ecology, soil pH, Streptophyta, terrestrial algae, ventaroles

\section{Introduction}

Debris fields are characterized by their unique air movement regime. In some cases, the cranny system of rock massive allows continual air circulation, resulting in the origin of summer ice holes and winter warm air exhalations, so-called ventaroles. The most famous ventarole locality in the Czech Republic is situated in the top parts of Boreč Hill (The České Středohoří Mts., Czech Republic). In winter, warm vapours rise from the top due to air circulation in the crevices. In spring and summer, the circulation is reversed and small ice stalagmites appear at the foot of the hill (Fig. 1). The differences in temperatures inside and outsides the ventaroles could reach $25^{\circ} \mathrm{C}$ (ANKERT 1917, КUвÁT 1971).

The Boreč Hill ventaroles are especially unique due to occurrence of several plant and animal species, intolerant of freezing temperatures. The locality is known as the only one recorded habitat of the Mediterranean liverwort Targionia hypophylla L. in the Czech Republic (PILous
1959). Rare plant, moss, and beetle species were recorded by several authors (LožEK 1954, KuBÁT 1971, Pujmanová 1990, NĚMcová 2001, RƯŽIČKA 2003). In this paper, the algal flora of ventaroles is reported for the first time. The main aims of this paper were 1) to compare the algal communities of the ventaroles and neighbouring areas, and 2) to ascertain the effect of the ventarole regime on the species composition and total diversity.

\section{Materials and Methods}

The research was realized in the top parts of Boreč Hill, in the south-western part of The České Středohoří Mts., the Czech Republic. Four sampling sites were allocated: two sites situated inside the ventaroles characterized by intense winter air exhalations, and two sites situated in the neighbouring area without winter exhalations:

1. A large ventarole near the information table No. 5, probably artificially enlarged in the 1950s (PILous 1959) $\left(50^{\circ} 30^{\prime} 51.3^{\prime \prime} \mathrm{N}, 1^{\circ} 59^{\prime} 19.4^{\prime \prime} \mathrm{E}, 443 \mathrm{~m}\right.$. a.s.1.). 
2. A small unaffected ventarole northwards to the site 1 (50³0'51.9” N, 1359'19.8” E, 441 m. a.s.1.).

3 . Area of $1 \mathrm{~m}^{2}$ without winter exhalations, southeastwards to the site $2\left(50^{\circ} 30^{\prime} 51.7^{\prime \prime} \mathrm{N}, 13^{\circ} 59^{\prime} 20.2^{\prime \prime}\right.$ E, 443 m. a.s.1.).

4. Area of $1 \mathrm{~m}^{2}$ without winter exhalations, northwestwards to the site $2\left(50^{\circ} 30^{\prime} 52.1^{\prime \prime} \mathrm{N}, 13^{\circ} 59^{\prime} 19.0^{\prime \prime}\right.$ E, 439 m. a.s.1.).

The localities were regularly investigated in three-month intervals (from December 2000 to February 2003). Three microbiotopes were sampled on each sampling date: "soil" (surface sample from the horizon of $0-2 \mathrm{~cm}$ ), "moss" (moss individuals or, in the winter, water squeezed from mosses growing in the ventaroles) and "rock" (mechanically scrapped rock surface). The samples were placed in sterile tubes and transported to the laboratory for analysis. Subsequently, the samples were both overfilled with liquid BBM medium (Bischoff \& Bold 1963) and the sample suspension was spread on Petri dishes with BBMagar. Algal microcolonies grown up after 8-10 weeks (at $15^{\circ} \mathrm{C}$ under daylight conditions) were isolated into unialgal cultures and cultivated at temperature $20^{\circ} \mathrm{C}$ under an illumination of $40 \mu \mathrm{mol} . \mathrm{m}^{-2} \cdot \mathrm{s}^{-1}$ provided by $18 \mathrm{~W}$ cool fluorescent tubes (Philips TLD 18W/33). To prepare diatom slides, the organic matter was removed by soaking in hydrogen peroxide $\left(\mathrm{H}_{2} \mathrm{O}_{2}\right.$; KRAMMER \& LANGe-Bertalot 1986). The frustules were dried on a coverslip and mounted in Pleurax resin. For a detailed investigation of diatom frustules and chrysophyte scales, drops were also dried onto formvar coated grids and examined with Philips 300 transmission electron microscope.

Individual species were identified using microscopic methods and Olympus BX51 light microscope with differential and phase contrasts and Olympus Z5060 microphotographic equipment. Identification was based on taxonomic monographs and reference books for terrestrial algae and cyanobacteria (Migula 1907, Geitler 1932, KorŠiKov 1953, Printz 1964, Prescott et al. 1972, Starmach 1972, EtTl 1978, 1983, RŮŽIČKA 1981, KOMÁREK \& FotT 1983, KRAMMER \& Lange-Bertalot 1986, 1988, 1991a, b, EtTl \& GÄRTNER 1988，1995, HINDÁK 1996, LENZENWEGER 1996, LoKHORSt 1996, ANDreEVA 1998, Krammer 2000, Lange-Bertalot 2001, Komárek \& Anagnostidis 2005). Formation of zoospores was stimulated by transferring unialgal cultures to tubes with both diluted BBM medium and fresh distilled water. The tubes were placed in darkness at a temperature of about $10^{\circ} \mathrm{C}$. Some of the unialgal cultures obtained were deposited either in the Culture Collection of Algae of Charles University in Prague (CAUP) or in the author's private culture collection.

Statistical analyses were carried out using the programme Canoco for Windows (TER BRAAK \& ŠMilauer 2002) and results of ordination were summarized using the programme CanoDraw (TER BRAAK \& ŠMILAUER 1998).

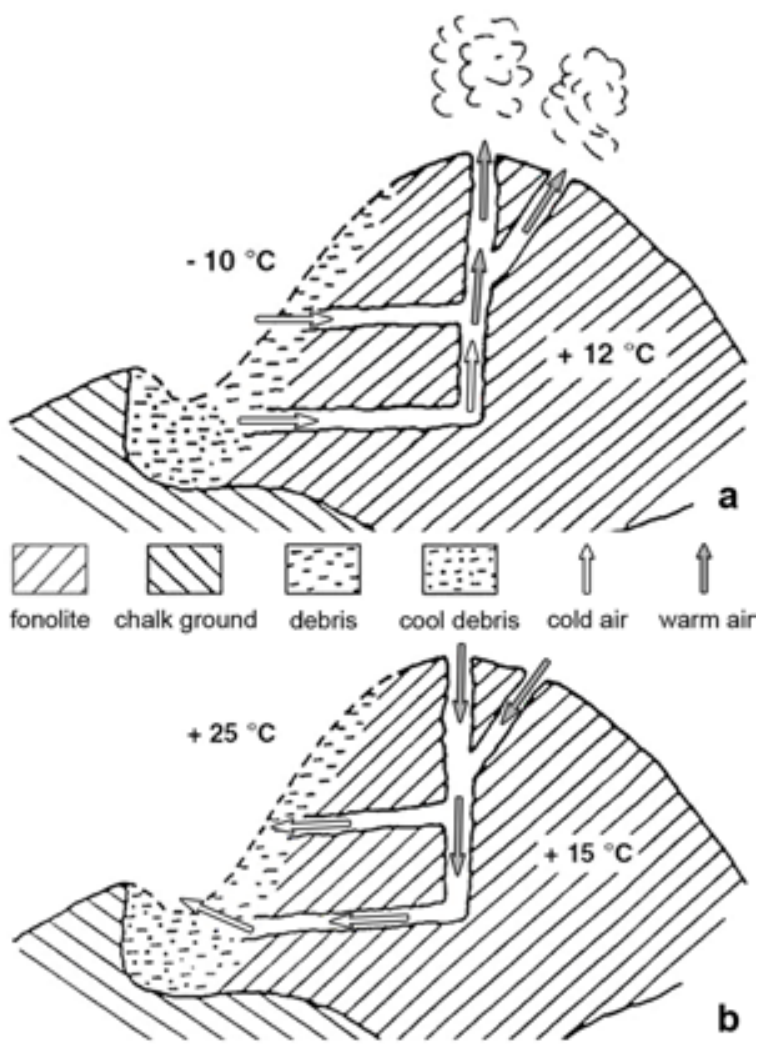

Fig. 1. Schematic drawing of the air circulation in the cranny system of rock massive in Boreč Hill: (a) winter air circulation; (b) summer air circulation (modified after KUČERA 1999).

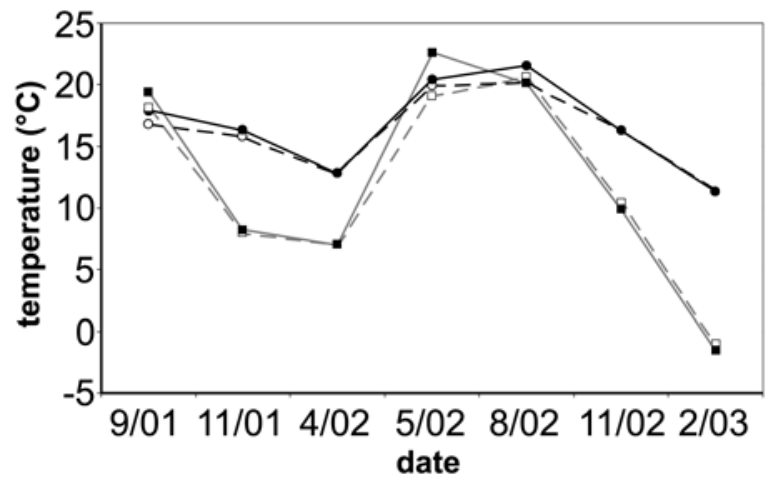

Fig. 2. Seasonal temperature fluctuation, measured in four investigated study sites (black circle, solid line - ventarole 1; white circle, dashed line - ventarole 2; white square, dashed line - sampling site 3; black square, solid line - sampling site 4). 


\section{Results and discussion}

\section{Analyses of environmental conditions}

Seasonal temperature fluctuation significantly differs between the ventaroles and unaffected study sites (Fig. 2). Whereas differences in temperature measured in the winter and summer reached $25^{\circ} \mathrm{C}$ in the unaffected sites, the temperature in the ventaroles oscillated only in the range of $10^{\circ} \mathrm{C}$. In the spring and summer, when the air circulation is reversed, the temperature was nearly identical in all the sites. However, during winter exhalations the ventaroles were distinctively warmer. The temperature of exhaled air was identical in both the ventaroles without reference to their size. A method of the continuous temperature measurement was used in 2003 to determine the lowest temperature that can occur in the ventaroles The lowest recorded temperature was $10.5^{\circ} \mathrm{C}$ (even acquired in the summer).

All four study sites are situated on phonolite, i.e. extrusive igneous rocks (lavas). Though the phonolite belongs to alkaline igneous rocks, measured soil $\mathrm{pH}$ had an acidic reaction (Fig. 3). This could be caused by several factors, e.g. human distractions like acidification and air pollution, rainwater leaching away basic ions, vegetation, or carbon dioxide from decomposing organic matter (TAMM \& HALLBÄCKEN 1986). Interestingly, obvious difference between the ventaroles and unaffected study sites was noted in soil $\mathrm{pH}$, which was higher in both the ventaroles (Fig. 3). Although $\mathrm{pH}$ values slightly fluctuated during the year, no seasonal changes were observed. The ventaroles are situated closely to the unaffected study sites. Accordingly, the difference in $\mathrm{pH}$ reaction has to be caused by the ventarole conditions. In contrast to the temperature difference caused by winter exhalations, the disparity in soil $\mathrm{pH}$ is difficult to interpret but has a principal biological importance. As $\mathrm{pH}$ levels drop below 5.5, the population of soil microbes changes and is dramatically reduced due to aluminium and manganese toxicity and reduced nutrient availability (MCFARLAND et al. 2001). By contrast, correct balance of trace elements is obtained where the soil $\mathrm{pH}$ is between 5.5 and 7.5. Therefore, the soil $\mathrm{pH}$ in the ventaroles $(\mathrm{pH} 4.7-$ 5.8) offers more favourable conditions for algal growth in comparison to the adjacent localities (pH 3.7-4.4).

\section{Species composition and diversity}

Algal diversity of the ventaroles greatly exceeded that found in the adjacent sampling sites. The ventaroles were inhabited by a markedly richer and more diversified algal flora. From a total of 112 species, 107 species of cyanobacteria and algae were recorded in the ventaroles, in contrast to 42 species found in their vicinity. Chrysophytes, eustigmatophytes and desmids occurred only in the ventaroles. Just 5 species were found outside the ventaroles. The list of species is given in Table 1. The species were investigated using morphological criteria only, without any molecular investigation. Even if purely morphological data are sufficient for standard ecological studies (where comparison of individual morphotypes illustrates influences of investigated factors), accurate description of the algal flora is essential for potential future comparisons. Since the recent molecular studies have shown a high hidden diversity of aero-terrestrial algae with little morphological differentiation (Huss et al. 1999, Krienitz et al. 2004, Sluiman et al. 2008), I place emphasis on a detailed morphological description of the investigated species. Hence, the majority of the investigated species are illustrated (Figs 4-8). Moreover, numerous micrographs of the detected cyanobacteria and algae have been published on the Web (ŠKaloud 2008).

The difficulties of current determination of green aerophytic algae could be well demonstrated by the strain Eutetramorus sp. This alga morphologically resembles species of genera

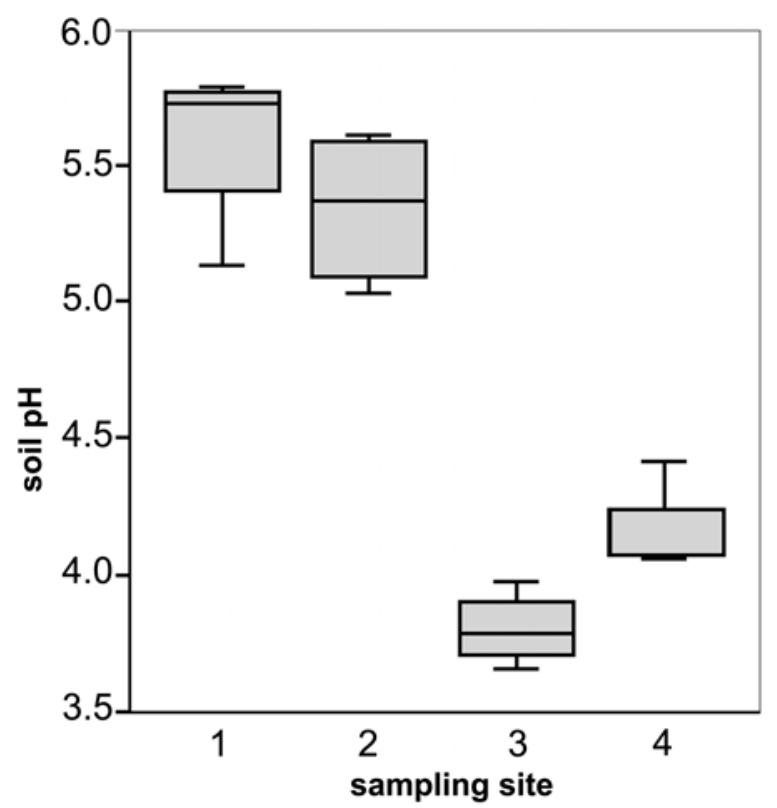

Fig. 3. Variability in the soil $\mathrm{pH}$ determined from five measurements in 2002 and 2003. 
Radiococcus SchmidLe or Eutetramorus WALTON (EtTl \& Gärtner 1995, KostiKov et al. 2002). However, whereas Radiococcus was determined to be a member of Chlorophyceae (Wolf et al. 2003), molecular investigation of isolated strains revealed that it belonged to Trebouxiophyceae (data not shown). On the other hand, Eutetramorus differs ecologically, as it has been so far recorded only from aquatic environments (GUIRY \& GUIRY 2008). Taxonomic designation is thus very difficult or even impossible for many algae and cyanobacteria included in this study. Therefore, the occurrences of particular morphospecies could be well used for demonstrating the algal diversity of studied sites. However, comparisons with other morphologically based studies on aero-terrestrial algae should be made very carefully, keeping in mind high cryptic diversity and low taxonomic significance of many morphological features defining these organisms.

\section{Ecological analyses}

On the PCA ordination plot, the samples from both the ventaroles form separate clusters, whereas the two adjacent localities are clustered together (Fig. 9). While the first ordination axis separates the ventaroles from the other two sampling sites, the second ordination axis clearly discriminates the ventaroles from one another. The first ordination axis obviously correlates with the species richness of particular samples. The samples with the greatest species richness were obtained from the moss samples in the ventarole 1 during winter exhalations (two rightmost samples in Fig. 9). The unique conditions inside the ventaroles thus seem to cause distinct increase in algal diversity.

To test the effect of the ventarole regime on the species composition and total diversity, RDA analysis was performed to determine if the localities are inhabited by different algae. The species composition of the ventaroles and adjacent localities was significantly different (p-value 0.0002). Similarly to PCA, the RDA analysis revealed the difference between both the ventaroles, in contrast to almost similar algal composition of the adjacent sampling sites (Fig. 10). Two species groups can be distinguished on the RDA ordination plot: $\boldsymbol{I}$ Species preferentially occurring in the ventarole 1 (e.g. Orthoseira CRAFWORD species, Kentrosphaera gibberosa and Euastrum brevisinuosum). II Species that were observed in both the ventaroles (e.g. Scenedesmus oocystiformis, Luticola mutica and Cosmarium obliquum var. trigonum). Whereas the group II encompasses species that can be indicated as ventarole-specific, the group $\boldsymbol{I}$ should comprehend species with affinity to some other environmental factors. The main difference between the ventaroles is their size and water regime. In contrast to the ventarole 2 , the ventarole 1 is much larger, enabling water condensation in moss plants during winter exhalations. The effect of water condensation on the species composition was thus tested by another RDA analysis (Fig. 11). The result was highly significant (p-value 0.0004), demonstrating a different algal composition inside wet mosses during the winter exhalations. Even if the effect of condensed water was tested against all the remaining samples (including rock and soil samples in the ventarole 1 during the exhalations), the first and second ordination axes well corresponded with the species groups $\boldsymbol{I}$ and II (Fig. 10), respectively. Thus, the larger species diversity in the ventarole 1 can be interpreted by seasonal formation of specific conditions, enabling short-term occurrence of several algal species preferring aquatic environments.

Due to high hidden cryptic diversity of several aero-terrestrial algal groups (e.g. cyanobacteria and coccal green algae), a precise comparison of their occurrences with respect to literary data is impossible. However, several algal species (in particular desmids and diatoms) can be used in this way owing to their good morphological circumscription and known ecological preferences. The diatom flora of the ventaroles resembles well the species composition found in the caves (GARBACKI et al. 1999). The most frequently occurred diatom genera, Diadesmis KützING, Hantzschia Grunow, Luticola MANN and Orthoseira, have been repeatedly noted from various caves worldwide (CARTER 1971, ST. Clair \& Rushforth 1976, PoulíčKová \& HaŠler 2007, Selvi \& Altuner 2007), including specific ice caves (LAURIOL et al. 2006). Moreover, specifically aquatic diatoms, Eunotia exigua, Navicula cryptocephala, and morphologically similar species of genera Frustulia RABENHORST and Synedra EHRENBERG, were reported from the crack cave in Ohio, the USA (DAYNER \& JOHANSEN 1991).

Contrary to diatoms, the desmid flora of the ventaroles is rather similar to algal communities found in ephemeral water bodies like temporary peat bog pools, wet or dripping rocks, etc. (RŮŽIČKA 1964, ETTL \& GÄRTNER 1995, 

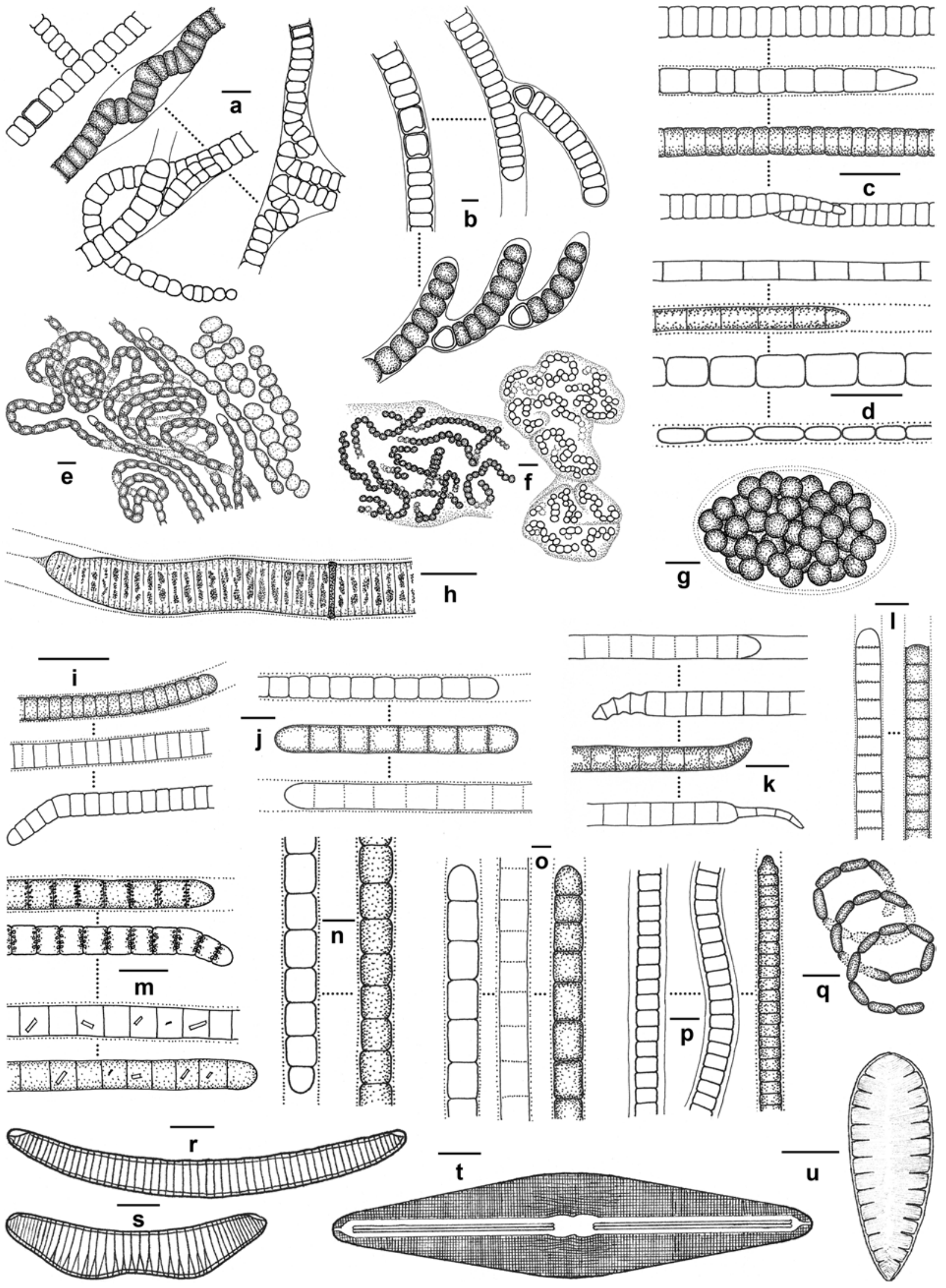

Fig. 4. (a) Fischerella thermalis; (b) Hassallia byssoidea; (c) Leptolyngbya foveolarum; (d) Leptolyngbya tenuis; (e) Nostoc calcicola; (f) Nostoc cf. edaphicum; (g) Nostoc sp.; (h) Oscillatoria cf. curviceps; (i) Phormidium cf. ambiguum; (j) Phormidium cf. animale; (k) Phormidium autumnale, (1) Phormidium sp. 1; (m) Phormidium sp. 2; (n) Phormidium sp. 3; (o) Phormidium sp. 4; (p) Phormidium sp. 5; (q) Pseudanabaena cf. catenata; (r) Eunotia bilunaris; (s) Eunotia implicata; (t) Frustulia saxonica; (u) Surirella cf. minuta. Scale bars $10 \mu \mathrm{m}$ (Figs a-b, f-m); $5 \mu \mathrm{m}$ (Figs c-d, n-u). 


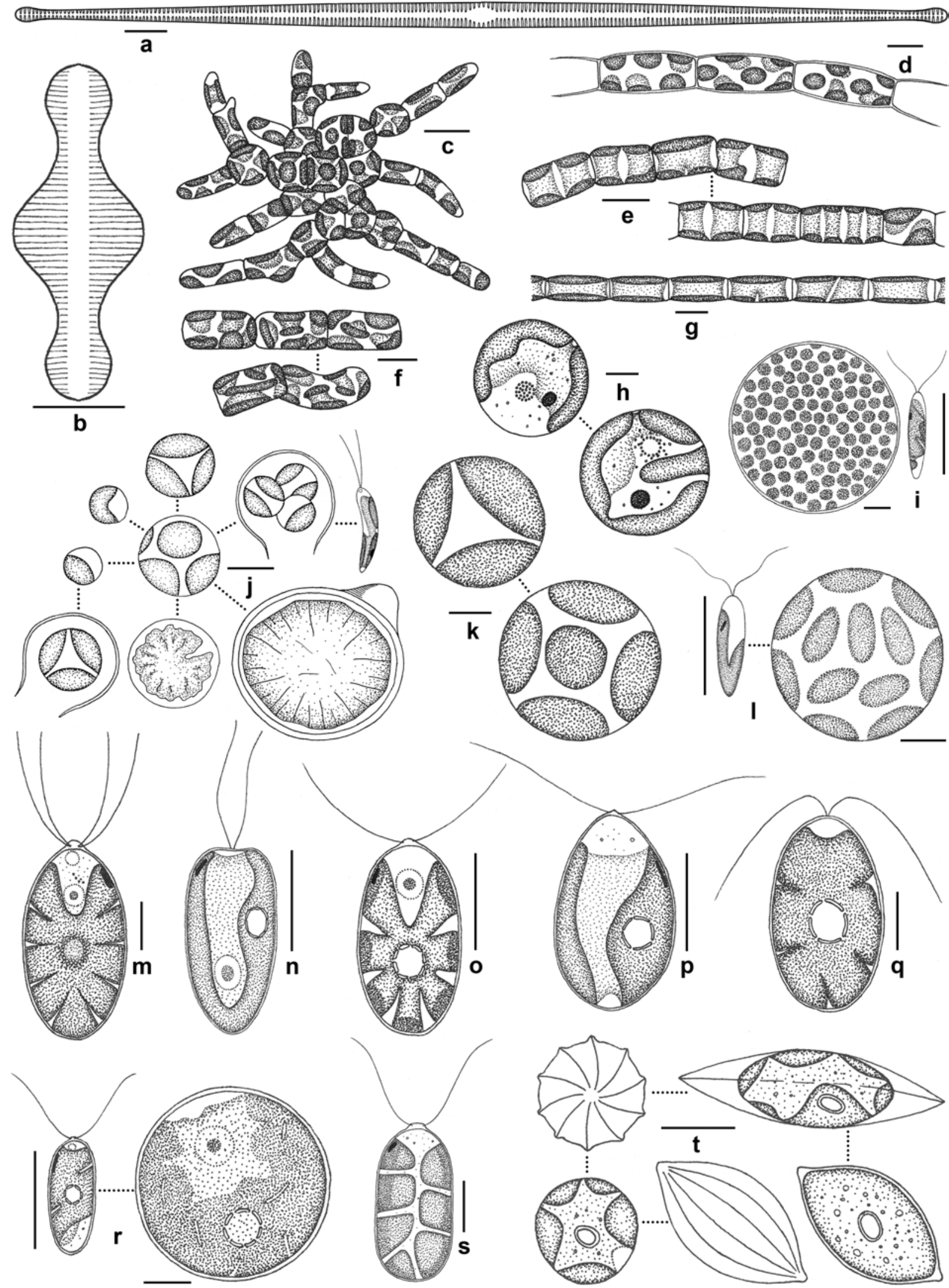

Fig. 5. (a) Synedra ulna; (b) Tabellaria flocculosa; (c) Heterococcus cf. crassulus; (d) Tribonema vulgare; (e) Xanthonema montanum; (f) Xanthonema solidum; (g) Xanthonema sp.; (h) Eustigmatos polyphem; (i) Bracteacoccus cf. grandis; (j) Bracteacoccus minor; (k) Bracteacoccus pseudominor, (1) Bracteacoccus sp.; (m) Carteria cf. klebsii; (n) Chlamydomonas callunae; (o) Chlamydomonas pseudintermedia; (p) Chlamydomonas $\mathrm{cf.}$ sestinensis; (q) Chlamydomonas sp.; (r) Chlorococcum infusionum; (s) Chloromonas rosae; (t) Coelastrella oocystiformis. Scale bars $10 \mu \mathrm{m}$ (Figs a, c, i); $5 \mu \mathrm{m}$ (Figs b, d-h, j-t). 

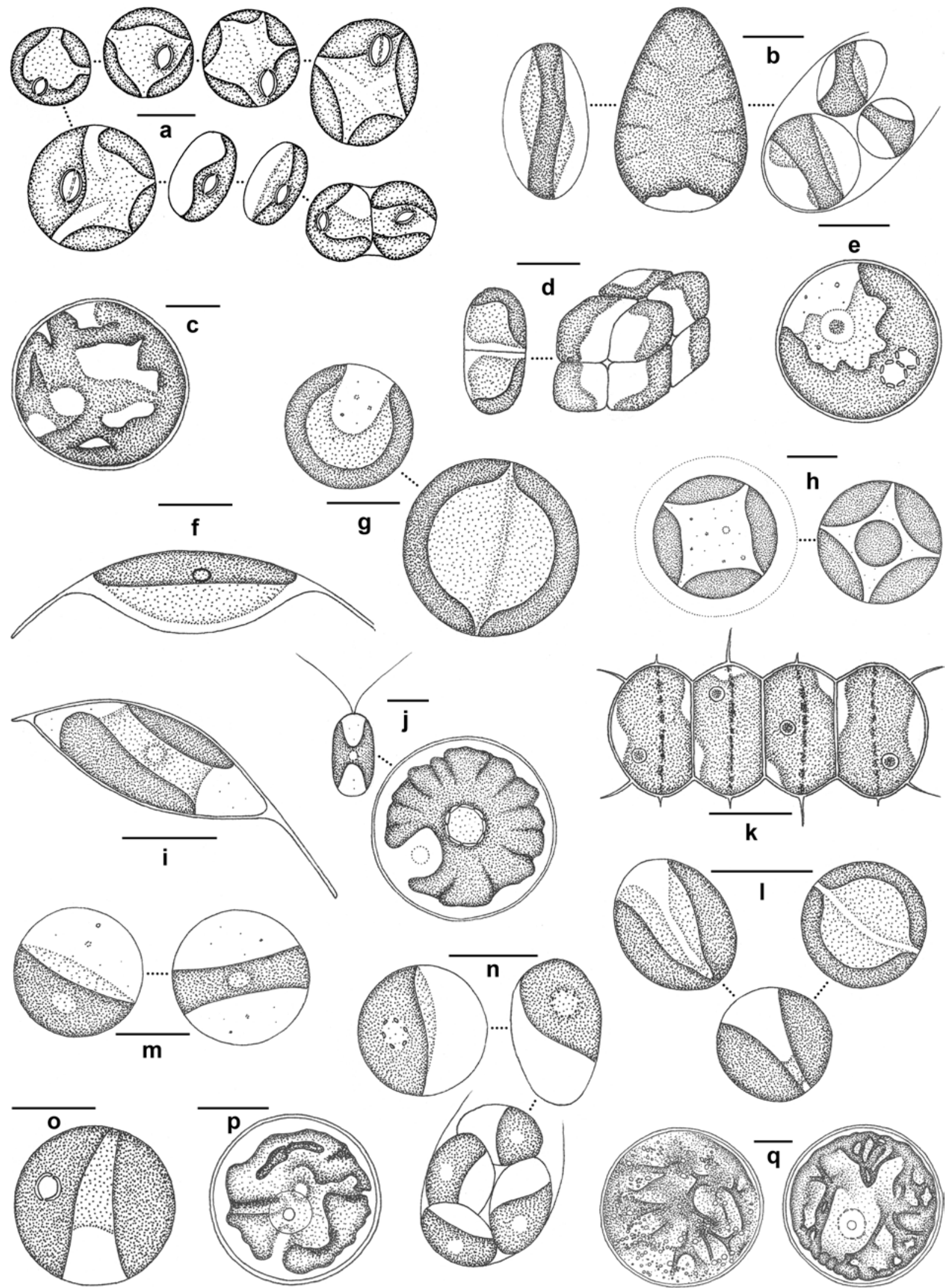

Fig. 6. (a) Coelastrella vacuolata; (b) Coleochlamys sp.; (c) Dictyochloris pulchra; (d) Diplosphaera chodatii; (e) Fasciculochloris boldii; (f) Keratococcus bicaudatus; (g) Mychonastes homosphaera; (h) Phacomyxa sp.; (i) Podohedra bicaudata; (j) Radiosphaera minuta; (k) Scenedemsus soli, (1) Auxenochlorella protothecoides; (m) 'Chlorella' cf. luteoviridis; (n) 'Chlorella' mirabilis; (o) Chlorella vulgaris; (p) Dictyochloropsis reticulate; (q) Dictyochloropsis splendida. Scale bar 5 $\mu \mathrm{m}$. 


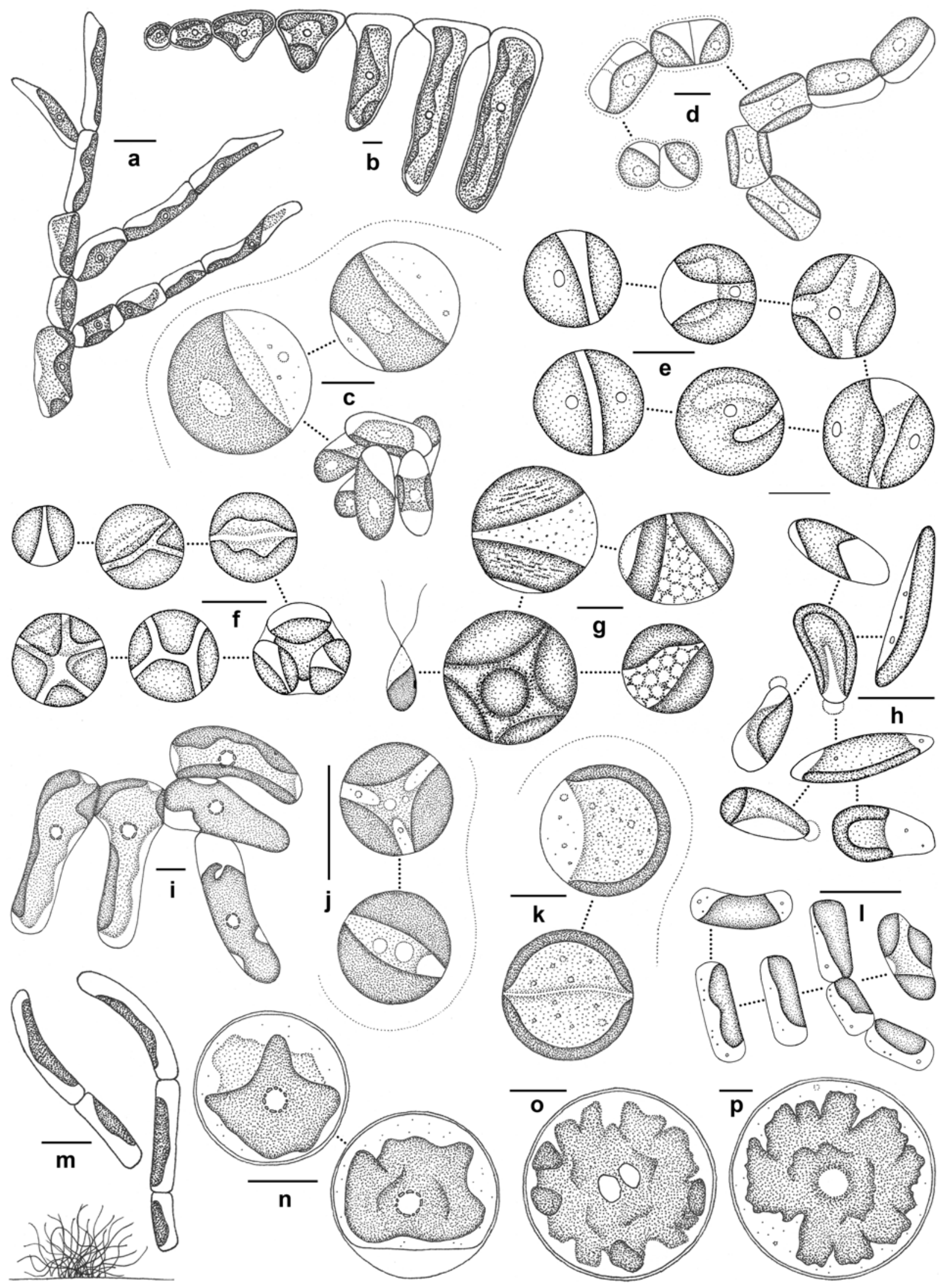

Fig. 7. (a) Dilabifilum printzii; (b) Dilabifilum sp.; (c) Eutetramorus sp.; (d) Geminella interrupta; (e) Lobosphaeropsis lobophora; (f) Muriella terrestris; (g) Myrmecia bisecta; (h) Pseudococcomyxa cf. simplex; (i) Rhexinema paucicellularis; (j) Schizochlamydella minutissima; (k) Schizochlamydella sp., (1) Stichococcus minor; (m) Stichococcus undulatus; (n) Trebouxia arboricola; (o) Trebouxia potteri; (p) Trebouxia sp. Scale bar $5 \mu \mathrm{m}$. 


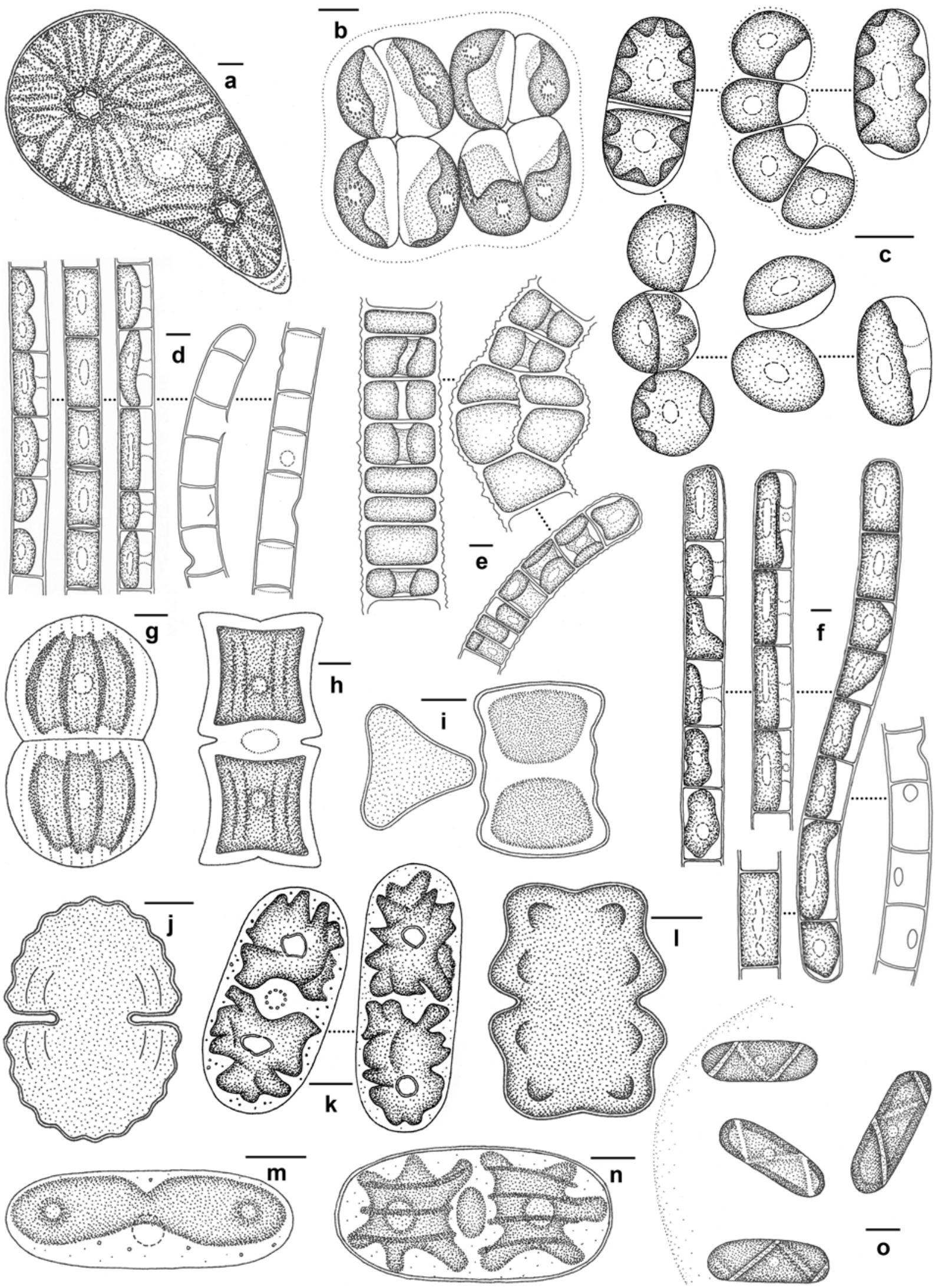

Fig. 8. (a) Kentrosphaera gibberosa; (b) Chlorokybus atmophyticus; (c) 'Geminella' terricola; (d) Klebsormidium flaccidum; (e) Klebsormidium mucosum; (f) Klebsormidium nitens; (g) Actinotaenium cucurbita; (h) Cosmarium decedens; (i) Cosmarium obliquum var. trigonum; (j) Cosmarium undulatum; (k) Cylindrocystis brebissonii; (1) Euastrum brevisinuosum; (m) Mesotaenium cf. endlicherianum; (n) Penium cf. spinospermum; (o) Spirotaenia endospira. Scale bar $5 \mu \mathrm{m}$. 

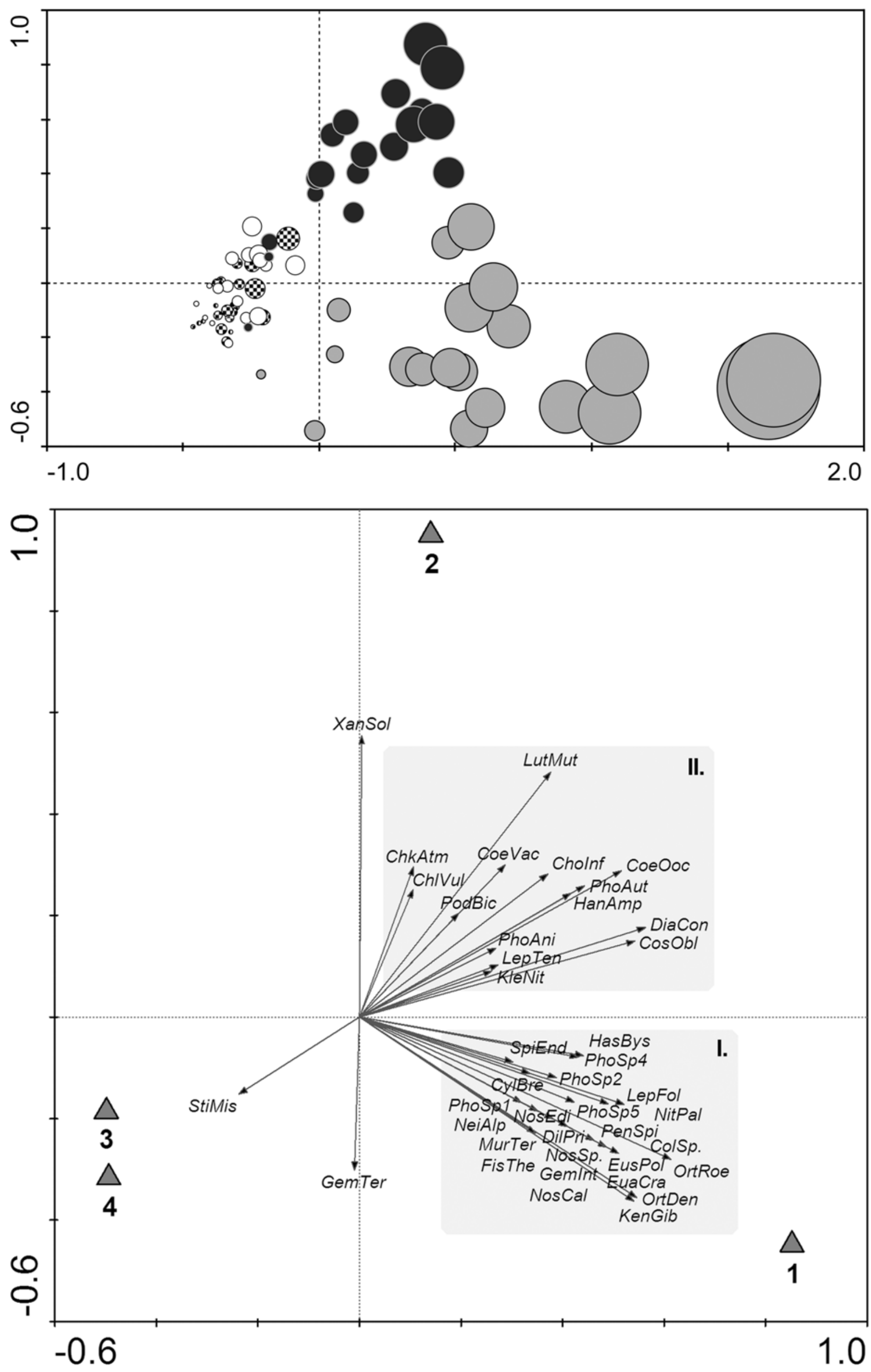


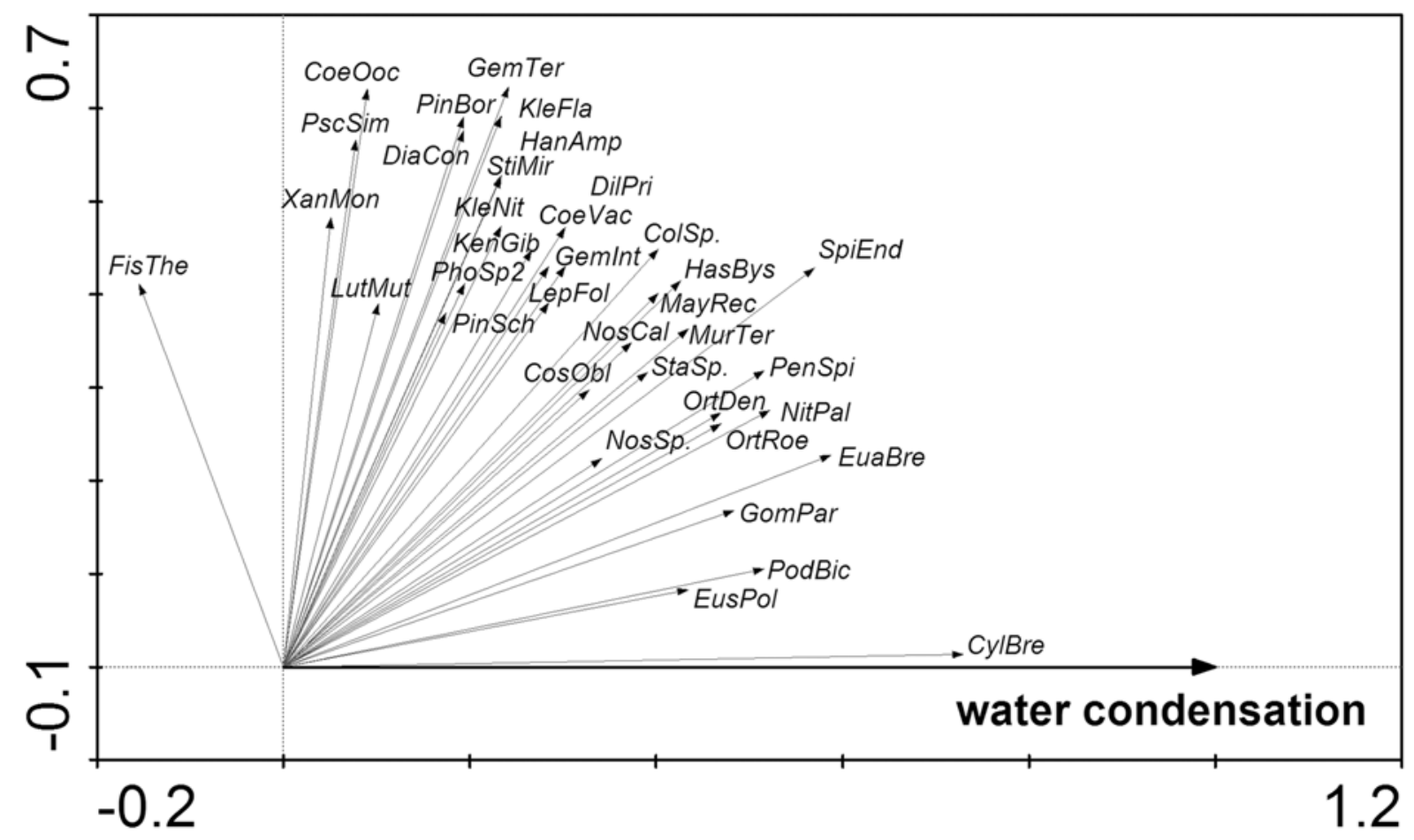

Fig. 11. The RDA ordination plot displaying preferences of the 37 best-fitted species to the moss samples saturated by condensed water during winter exhalations in the ventaroles. For the species acronyms see Table 1.

Fig. 9. The PCA ordination plot of the samples (grey - ventarole 1; black - ventarole 2; white - sampling site 3; chessboard sampling site 4). Symbol sizes correspond with the species richness of the particular sample.

Fig. 10. The RDA ordination plot displaying preferences of the 41 best-fitted species to four sampling sites: $(1,2)$ ventaroles; $(3,4)$ adjacent sampling sites. I species preferentially occurring only in the large ventarole (sampling site 1), II species occurring in both the ventaroles. For the species acronyms see Table 1 .

Table 1. List of species with "total occurrence" values (sums of species occurrences for the set of samples) in localities (1-4), microbiotopes [(m) moss; (r) rock; (s) soil)] and in relation to warm, moist winter exhalations (+ present, - absent; occurrences counted for the ventaroles only).

\begin{tabular}{|c|c|c|c|c|c|c|c|c|c|c|c|}
\hline & \multirow[b]{2}{*}{ Acronym } & \multirow[b]{2}{*}{ Figure } & \multicolumn{4}{|c|}{ Localities } & \multicolumn{3}{|c|}{ Microbiotopes } & \multicolumn{2}{|c|}{ Exhalations } \\
\hline & & & 1 & 2 & 3 & 4 & $\mathbf{m}$ & $\mathbf{r}$ & $\mathbf{s}$ & + & - \\
\hline \multicolumn{12}{|l|}{ Cyanobacteria } \\
\hline Fischerella thermalis SCHWABE ex GOMONT & FisThe & $4 a$ & 7 & - & - & - & 2 & 6 & 3 & 4 & 2 \\
\hline Hassallia byssoidea Hassall ex Bornet et Flahault & HasBys & $4 b$ & 8 & 3 & 1 & - & 7 & 7 & 3 & 7 & 4 \\
\hline $\begin{array}{l}\text { Leptolyngbya foveolarum (RABENHORST ex GOMONT) } \\
\text { ANAGNOSTIDIS et KOMÁREK }\end{array}$ & LepFol & $4 \mathrm{c}$ & 9 & 3 & - & 1 & 8 & 6 & 5 & 5 & 4 \\
\hline $\begin{array}{l}\text { Leptolyngbya tenuis (GOMONT) ANAGNOSTIDIS et } \\
\text { KOMÁREK }\end{array}$ & LepTen & $4 \mathrm{~d}$ & 7 & 6 & 2 & 3 & 6 & 9 & 9 & 8 & 4 \\
\hline Nostoc calcicola BréBISSON ex Bornet et FlaHault & $\mathrm{NosCal}$ & $4 \mathrm{e}$ & 5 & - & - & - & 4 & 2 & 5 & 3 & 2 \\
\hline Nostoc cf. edaphicum KondRateva & NosEdi & $4 \mathrm{f}$ & 6 & - & - & - & 1 & - & 5 & 3 & 2 \\
\hline Nostoc sp. & NosSp. & $4 g$ & 5 & - & - & - & 3 & 4 & - & 3 & 1 \\
\hline Oscillatoria cf. curviceps AGARDH ex GOMONT & - & $4 \mathrm{~h}$ & 1 & - & - & - & - & - & 1 & - & 1 \\
\hline Phormidium cf. ambiguum GoмоNт & - & $4 \mathrm{i}$ & 1 & - & - & - & - & - & 1 & 1 & - \\
\hline $\begin{array}{l}\text { Phormidium cf. animale (AGARDH ex GOMONT) } \\
\text { ANAGNOSTIDIS et KOMÁREK }\end{array}$ & PhoAni & $4 j$ & 4 & 5 & - & - & 7 & 3 & - & 2 & 5 \\
\hline Phormidium autumnale AGARDH ex GOMONT & PhoAut & $4 \mathrm{k}$ & 8 & & 1 & - & 15 & 8 & 5 & 8 & 6 \\
\hline Phormidium sp. 1 & PhoSpl & 41 & 5 & - & - & - & 1 & - & 4 & 2 & 3 \\
\hline
\end{tabular}




\begin{tabular}{|c|c|c|c|c|c|c|c|c|c|c|c|}
\hline & \multicolumn{6}{|c|}{ Localities } & \multicolumn{3}{|c|}{ Microbiotopes } & \multicolumn{2}{|c|}{ Exhalations } \\
\hline & Acronym & Figure & 1 & 2 & 3 & 4 & m & $\mathbf{r}$ & $\mathbf{s}$ & + & - \\
\hline Phormidium sp. 2 & PhoSp2 & $4 \mathrm{~m}$ & 8 & 3 & - & 2 & 5 & 5 & 6 & 3 & 5 \\
\hline Phormidium sp. 3 & - & $4 n$ & - & 2 & - & - & - & - & 2 & - & - \\
\hline Phormidium sp. 4 & PhoSp4 & 40 & 7 & 2 & - & - & 6 & 2 & 5 & 3 & 3 \\
\hline Phormidium sp. 5 & PhoSp 5 & $4 p$ & 6 & 1 & - & - & 5 & 3 & 2 & 4 & 2 \\
\hline Pseudanabaena cf. catenata LAUTERBORN & - & $4 q$ & - & 1 & - & - & - & 1 & - & - & 1 \\
\hline \multicolumn{12}{|l|}{ Bacillariophyceae } \\
\hline Aulacoseira granulata (EHRENBERG) SIMONSEN & - & - & 1 & 1 & - & - & 2 & - & - & 1 & 1 \\
\hline Cocconeis placentula EHRENBERG & - & - & 1 & - & - & - & 1 & - & - & 1 & - \\
\hline Diadesmis contenta (Grunow ex VAN Heurck) ManN & DiaCon & - & 9 & 9 & 3 & 3 & 18 & 12 & 16 & 8 & 6 \\
\hline Eunotia bilunaris (EHRENBERG) Mills & - & $4 \mathrm{r}$ & - & 1 & - & - & - & - & 1 & 1 & - \\
\hline Eunotia implicata NöRPel, LANGe-Bertalot et Alles & - & $4 s$ & 1 & - & - & - & 1 & - & - & 1 & - \\
\hline Frustulia saxonica RABENHORST & - & $4 \mathrm{t}$ & - & - & - & 2 & 1 & 1 & - & - & - \\
\hline Gomphonema parvulum (KÜTZING) VAN HEURCK & GomPar & - & 2 & 1 & 1 & - & 1 & 2 & 3 & 1 & - \\
\hline Hantzschia amphioxys (EHRENBERG) GRUNOW & HanAmp & - & & & 5 & 3 & 18 & 15 & 13 & 8 & 8 \\
\hline Kobayasiella sp. & - & - & 3 & 2 & - & - & 1 & - & 4 & 2 & 2 \\
\hline Luticola mutica (KÜTZING) MANN & LutMut & - & 7 & & 1 & 1 & 13 & 8 & 13 & 8 & 6 \\
\hline Mayamaea recondita (Hustedt) LANGE-Bertalot & MayRec & - & 4 & 3 & 5 & 7 & 7 & 4 & 13 & 5 & 1 \\
\hline $\begin{array}{l}\text { Navicula oligotraphenta LANGE-BERTALOT et } \\
\text { HofmanN }\end{array}$ & - & - & 1 & - & - & - & 1 & - & - & 1 & - \\
\hline Neidium alpinum HustedT & NeiAlp & - & 5 & - & - & - & - & - & 5 & 2 & 3 \\
\hline Nitzschia palea (KÜTZING) W. SмIтH & NitPal & - & 9 & 2 & 1 & - & 7 & 5 & 8 & 4 & 3 \\
\hline $\begin{array}{l}\text { Orthoseira dendroteres (EHRENBERG) CRAWFORD, } \\
\text { HaW. et Kelly }\end{array}$ & OrtDen & - & 9 & - & - & 3 & 10 & 9 & 2 & 4 & 3 \\
\hline Orthoseira roeseana (RABENHORST) O`MEARA & OrtRoe & - & & 1 & - & - & 9 & 8 & 1 & 5 & 4 \\
\hline Pinnularia borealis EHRENBERG & PinBor & - & & & 7 & 8 & 26 & 16 & 25 & 8 & 8 \\
\hline Pinnularia schoenfelderi KRAMMER & PinSch & - & 4 & 5 & - & 2 & 4 & 4 & 7 & 5 & 4 \\
\hline Pinnularia sudetica (HILSE) HILSE & - & - & 1 & - & - & - & - & - & 1 & - & 1 \\
\hline Stauroneis sp. & StaSp. & - & 5 & 2 & - & 2 & 4 & 2 & 3 & 3 & 2 \\
\hline Surirella cf. minuta BRÉBISSON & - & $4 u$ & 1 & - & - & - & - & - & 1 & - & 1 \\
\hline Synedra ulna (Nitzsch) EHRENBERG & - & $5 \mathrm{a}$ & 1 & - & - & - & 1 & - & - & 1 & - \\
\hline Tabellaria flocculosa (Rотн) KÜтZING & - & $5 b$ & 2 & - & 2 & - & 2 & - & 2 & 2 & - \\
\hline \multicolumn{12}{|l|}{ Chrysophyceae } \\
\hline Paraphysomonas vestita (StOKes) De SADELEER & - & - & 1 & - & - & - & 1 & - & - & 1 & - \\
\hline Spiniferomonas sp. & - & - & 1 & - & - & - & 1 & - & - & 1 & - \\
\hline \multicolumn{12}{|l|}{ Synurophyceae } \\
\hline $\begin{array}{l}\text { Synura multidentata (BALONOv et KUZMIN) PéTERFI et } \\
\text { MoMeU }\end{array}$ & - & - & 1 & - & - & - & - & 1 & - & - & - \\
\hline \multicolumn{12}{|l|}{ Xanthophyceae } \\
\hline Heterococcus cf. crassulus VISCHER & - & $5 \mathrm{c}$ & 1 & - & - & - & - & - & 1 & 1 & - \\
\hline Tribonema vulgare PASCHER & - & $5 d$ & 3 & 1 & - & - & - & 1 & 3 & 3 & 1 \\
\hline Xanthonema montanum (VISCHER) SILVA & XanMon & $5 e$ & 4 & 7 & 2 & 5 & 10 & 7 & 13 & 5 & 5 \\
\hline Xanthonema solidum (VISCHER) SILVA & XanSol & $5 \mathrm{f}$ & - & 8 & 4 & - & 7 & 5 & 7 & 3 & 3 \\
\hline Xanthonema sp. & - & $5 \mathrm{~g}$ & 1 & - & - & - & - & - & 1 & 1 & - \\
\hline \multicolumn{12}{|l|}{ Eustigmatophyceae } \\
\hline Eustigmatos polyphem (PITSCHMANN) HiBBERD & EusPol & $5 \mathrm{~h}$ & 6 & - & - & - & 3 & 2 & 5 & 4 & 1 \\
\hline \multicolumn{12}{|l|}{ Chlorophyceae } \\
\hline Bracteacoccus cf. grandis BISCHOFF et BoLD & - & $5 i$ & 1 & 2 & 2 & - & 2 & 1 & 3 & 2 & 1 \\
\hline Bracteacoccus minor (CHODAT) Petrova & - & $5 \mathrm{j}$ & 3 & - & - & - & - & 1 & 2 & 3 & - \\
\hline
\end{tabular}




\begin{tabular}{|c|c|c|c|c|c|c|c|c|c|c|c|}
\hline & \multirow[b]{2}{*}{ Acronym } & \multirow[b]{2}{*}{ Figure } & \multicolumn{4}{|c|}{ Localities } & \multicolumn{3}{|c|}{ Microbiotopes } & \multicolumn{2}{|c|}{ Exhalations } \\
\hline & & & 1 & 2 & 3 & 4 & $\mathbf{m}$ & $\mathbf{r}$ & $\mathbf{s}$ & + & - \\
\hline Bracteacoccus pseudominor BISCHOFF et BOLD & - & $5 \mathrm{k}$ & - & - & 1 & - & - & - & 1 & - & - \\
\hline Bracteacoccus sp. & - & 51 & 2 & 3 & - & - & 3 & 1 & 2 & 4 & 1 \\
\hline Carteria cf. klebsii (DANGEARD) FrancÉ & - & $5 \mathrm{~m}$ & 3 & - & - & - & 3 & - & 1 & 3 & - \\
\hline Chlamydomonas callunae ЕттL & - & $5 n$ & - & 2 & - & - & 1 & - & 1 & 2 & - \\
\hline Chlamydomonas pseudintermedia BEHRE et SCHWABE & - & 50 & - & 1 & - & - & 1 & - & - & 1 & - \\
\hline Chlamydomonas cf. sestinensis GERLOFF & - & $5 p$ & 2 & - & - & - & 2 & - & - & 2 & - \\
\hline Chlamydomonas sp. & - & $5 q$ & - & - & 2 & 1 & - & - & 3 & - & - \\
\hline Chlorococcum infusionum (Schrank) MENEGHINI & ChoInf & $5 \mathrm{r}$ & 6 & 9 & 1 & 1 & 9 & 7 & 11 & 5 & 6 \\
\hline Chloromonas rosae (ЕттL) ЕтTL & - & $5 \mathrm{~s}$ & 1 & - & - & - & 1 & - & - & 1 & - \\
\hline $\begin{array}{l}\text { Coelastrella oocystiformis (LuND) HEGEWALD et } \\
\text { HANAGATA }\end{array}$ & $\mathrm{CoeOoc}$ & $5 \mathrm{t}$ & 9 & 9 & - & 1 & 11 & 13 & 10 & 7 & 7 \\
\hline $\begin{array}{l}\text { Coelastrella vacuolata (ShiHIRA et Krauss) } \\
\text { HegeWald et Hanagata }\end{array}$ & CoeVac & $6 \mathrm{a}$ & 4 & 5 & - & - & 6 & 5 & 5 & 6 & 3 \\
\hline Coleochlamys sp. & ColSp. & $6 b$ & 6 & - & - & - & 5 & 5 & - & 3 & 3 \\
\hline Dictyochloris pulchra DeAsOn et HERNDON & - & $6 c$ & 2 & - & - & - & 1 & 1 & - & 1 & 1 \\
\hline Diplosphaera chodatii BIALOSUKNIA & - & $6 \mathrm{~d}$ & 2 & - & - & 1 & 2 & 1 & - & 2 & - \\
\hline Fasciculochloris boldii McLeAn et TraINOR & - & $6 e$ & - & 1 & - & - & - & - & 1 & 1 & - \\
\hline Keratococcus bicaudatus (BRAun) J.B. Petersen & - & $6 f$ & 4 & - & - & - & 3 & - & 1 & 2 & 1 \\
\hline $\begin{array}{l}\text { Mychonastes homosphaera (SKUJA) KALINA et } \\
\text { PUnČOCHÁŘ́VÁ }\end{array}$ & - & $6 \mathrm{~g}$ & 1 & - & 1 & - & - & 1 & 1 & 1 & - \\
\hline Phacomyxa sp. & - & $6 \mathrm{~h}$ & - & 1 & - & - & - & - & 1 & 1 & - \\
\hline Podohedra bicaudata GeITLER & PodBic & $6 \mathrm{i}$ & 3 & 4 & - & - & 6 & - & 2 & 4 & 2 \\
\hline Radiosphaera minuta HERNDON & - & $6 \mathrm{j}$ & 1 & - & - & - & - & 1 & - & - & - \\
\hline Scenedemsus soli НоRTOBAGYI & - & $6 \mathrm{k}$ & 1 & - & - & - & - & 1 & - & - & - \\
\hline \multicolumn{12}{|l|}{ Trebouxiophyceae } \\
\hline $\begin{array}{l}\text { Auxenochlorella protothecoides (KRÜGER) KaLINA et } \\
\text { PUnČOCHÁŘ́vÁ }\end{array}$ & - & 61 & 2 & - & - & - & 1 & 2 & 1 & 1 & 1 \\
\hline ,Chlorella' cf. luteoviridis СНОDАТ & - & $6 \mathrm{~m}$ & 3 & - & - & 2 & 3 & 1 & 1 & 1 & 1 \\
\hline ,Chlorella' minutissima FotT et NovÁková & - & - & 2 & 3 & - & - & 2 & - & 3 & 4 & 1 \\
\hline ,Chlorella' mirabilis ANDREEVA & - & $6 n$ & 2 & 1 & 1 & - & 3 & - & 1 & - & 2 \\
\hline Chlorella vulgaris BEIJERINCK & ChlVul & 60 & 1 & 3 & - & - & 3 & - & 2 & 3 & - \\
\hline Choricystis minor (SKUנA) FотT & - & - & - & 3 & - & - & 1 & 2 & - & 2 & 1 \\
\hline $\begin{array}{l}\text { Dictyochloropsis reticulata (TsCHERMAK-WoEss) } \\
\text { TSCHERMAK-WOESS }\end{array}$ & - & $6 \mathrm{p}$ & - & 1 & 1 & 1 & 2 & - & 1 & - & 1 \\
\hline $\begin{array}{l}\text { Dictyochloropsis splendida (GeITLER) TSCHERMAK- } \\
\text { WoEss }\end{array}$ & - & $6 \mathrm{q}$ & - & 1 & 2 & 1 & 2 & 3 & 1 & 1 & - \\
\hline Dilabifilum printzii (VISCHER) TSCHERMAK-WOESS & DilPri & $7 \mathrm{a}$ & 4 & - & - & - & 3 & 3 & 1 & 3 & 1 \\
\hline Dilabifilum sp. & - & $7 b$ & 2 & - & - & - & 1 & 2 & - & 2 & - \\
\hline Eutetramorus sp. & - & $7 \mathrm{c}$ & 2 & 1 & - & - & - & - & 3 & 3 & - \\
\hline Geminella interrupta (TURPIN) LAGERHEIM & GemInt & $7 \mathrm{~d}$ & 5 & - & - & - & 2 & 5 & 1 & 4 & - \\
\hline $\begin{array}{l}\text { Lobosphaeropsis lobophora (ANDREEVA) ETTL et } \\
\text { GÄRTNER }\end{array}$ & - & $7 e$ & 1 & 1 & - & 1 & 1 & - & 2 & - & - \\
\hline Muriella terrestris J.B. PETERSEN & MurTer & $7 f$ & 6 & - & 2 & - & 3 & 4 & 4 & 4 & 2 \\
\hline Myrmecia bisecta ReISIGL & - & $7 \mathrm{~g}$ & 1 & 6 & 5 & 1 & 7 & 3 & 5 & 5 & 2 \\
\hline Pseudococcomyxa cf. simplex (MAINX) FotT & PscSim & $7 \mathrm{~h}$ & 3 & 5 & 6 & 4 & 15 & 13 & 13 & 5 & 3 \\
\hline Rhexinema paucicellularis (VISCHER) GEITLER & - & $7 \mathrm{i}$ & - & 1 & - & - & - & 1 & - & 1 & - \\
\hline Schizochlamydella minutissima BROADY & - & $7 \mathrm{j}$ & 1 & 2 & - & - & 1 & 1 & 2 & 3 & - \\
\hline Schizochlamydella sp. & - & $7 \mathrm{k}$ & 2 & - & - & - & 2 & - & - & 2 & - \\
\hline Stichococcus minor NÄGELI & StiMir & 71 & 6 & 5 & 5 & 6 & 17 & 13 & 8 & 6 & 4 \\
\hline Stichococcus minutus Grintzesco et PÉTERFI & StiMis & - & - & - & - & 5 & 2 & 4 & 2 & - & - \\
\hline Stichococcus undulatus VinATZER & - & $7 \mathrm{~m}$ & 1 & - & - & - & 1 & 1 & - & 1 & - \\
\hline Trebouxia arboricola De Puymaly & - & $7 \mathrm{n}$ & - & - & 2 & - & - & 2 & 1 & - & - \\
\hline
\end{tabular}




\begin{tabular}{|c|c|c|c|c|c|c|c|c|c|c|c|}
\hline & \multirow[b]{2}{*}{ Acronym } & \multirow[b]{2}{*}{ Figure } & \multicolumn{4}{|c|}{ Localities } & \multicolumn{3}{|c|}{ Microbiotopes } & \multicolumn{2}{|c|}{ Exhalations } \\
\hline & & & 1 & 2 & 3 & 4 & $\mathbf{m}$ & $\mathbf{r}$ & $\mathbf{s}$ & + & - \\
\hline Trebouxia potteri AHMADJIAN ex GÄRTNER & - & 70 & - & 2 & - & - & 2 & - & - & 2 & - \\
\hline Trebouxia sp. & - & $7 \mathrm{p}$ & 1 & - & - & - & 1 & - & - & 1 & - \\
\hline \multicolumn{12}{|l|}{ Ulvophyceae } \\
\hline Kentrosphaera gibberosa VODENIČAROv et BENDERLIEV & KenGib & $8 \mathrm{a}$ & & - & 2 & 2 & 7 & 8 & 8 & 4 & 4 \\
\hline \multicolumn{12}{|l|}{ Chlorokybophyceae } \\
\hline Chlorokybus atmophyticus GeITLER & ChkAtm & $8 \mathrm{~b}$ & 1 & 5 & - & - & 3 & 3 & - & 3 & 1 \\
\hline \multicolumn{12}{|l|}{ Klebsormidiophyceae } \\
\hline , Geminella 'terricola J.B. Petersen & GemTer & $8 \mathrm{c}$ & 4 & - & 5 & 4 & 8 & 9 & 8 & 2 & 2 \\
\hline $\begin{array}{l}\text { Klebsormidium flaccidum (KÜtZING) Silva, Mattox } \\
\text { et BlaCKwELl }\end{array}$ & KleFla & $8 \mathrm{~d}$ & 8 & 8 & 9 & 8 & 25 & 23 & 22 & 8 & 5 \\
\hline Klebsormidium mucosum (J.B. Petersen) LOKHORST & - & $8 \mathrm{e}$ & 1 & 3 & 2 & - & 4 & 3 & - & 1 & 3 \\
\hline Klebsormidium nitens (MENEGHINI) LOKHORST & KleNit & $8 \mathrm{f}$ & 9 & & 8 & 5 & 18 & 19 & 14 & 8 & 8 \\
\hline \multicolumn{12}{|l|}{ Zygnematophyceae } \\
\hline Actinotaenium cucurbita (BRÉBISSON) TeILING & - & $8 \mathrm{~g}$ & 2 & - & - & - & 2 & - & - & 2 & - \\
\hline Cosmarium decedens (REINSCH) RACIBORSKI & - & $8 \mathrm{~h}$ & 1 & - & - & - & 1 & - & - & 1 & - \\
\hline $\begin{array}{l}\text { Cosmarium obliquum NORDSTEDT var. trigonum W. } \\
\text { WeST }\end{array}$ & $\mathrm{Cos} \mathrm{Obl}$ & $8 \mathrm{i}$ & & 9 & - & - & 15 & 11 & 2 & 8 & 7 \\
\hline Cosmarium undulatum CORDA ex RALFs & - & $8 \mathrm{j}$ & 1 & - & - & - & - & 1 & - & - & - \\
\hline $\begin{array}{l}\text { Cylindrocystis brebissonii (MENEGHINI ex RALFS) de } \\
\text { BARY }\end{array}$ & CylBre & $8 \mathrm{k}$ & 4 & 1 & - & - & 4 & 3 & - & 4 & 1 \\
\hline Euastrum brevisinuosum (NORDSTEDT) KOUWETS & EuaBre & 81 & 9 & - & - & - & 7 & 5 & - & 4 & 3 \\
\hline Mesotaenium cf. endlicherianum NäGELI & - & $8 \mathrm{~m}$ & 3 & - & - & - & 3 & - & - & 3 & - \\
\hline Penium cf. spinospermum JosHUA & PenSpi & $8 n$ & 5 & - & - & - & 4 & 4 & - & 4 & - \\
\hline Spirotaenia endospira (BRÉBISSON) ARCHER & SpiEnd & 80 & 3 & 1 & - & - & 4 & 2 & - & 4 & - \\
\hline
\end{tabular}

Williamson 2000, ŠŤAstnÝ 2008). Actinotaenium cucurbita, Cylindrocystis brebissonii, Cosmarium undulatum and Mesotaenium NäGELI species are commonly mentioned from aero-terrestrial substrata, having strong resistance to long-term desiccation (ETTL \& GÄRTNER 1995, WILLIAMSON 2000). None of the observed desmid taxa could be indicated as an entirely aquatic species, though some of them are occasionally reported from aquatic environments (e.g. Penium spinospermum from acid boggy water bodies; KRIEGER 1937). Interestingly, even if several investigated taxa can be designated as extremely rare species (e.g. Cosmarium obliquum, var. trigonum, Euastrum brevisinuosum, Spirotaenia endospira), they were repeatedly found in high abundances during the winter exhalations. For example, mucilaginous algal mats of Cosmarium obliquum, var. trigonum were macroscopically visible in winter season inside the ventarole 1.

To summarize, the Boreč Hill ventaroles represent a unique type of biotope, with a specific algal flora adapted to the periodical periods of warm, moist air exhalations of several months' duration. As a whole, the algal composition could not be assigned to any known aero-terrestrial biotope. Rather, they can be considered as a specific type of transitional biotope between aero-terrestrial and freshwater environments. Moreover, the ventaroles are demonstrably inhabited by several very rare species. Thus, the Boreč Hill ventaroles definitely represent one of the most valuable aero-terrestrial localities in the region.

\section{Acknowledgements}

The author would like to express his deep thanks to Jiři Neustupa for his valuable comments and various suggestions. I would also like to thank Tomáš Kalina, Alena Lukešová, Jiří Komárek, Jana Veselá and Jan Št'astný for their help with species determination. 


\section{References}

AndreEva, V.M. (1998): Poczvennye i aerophilnye zelyonye vodorosli (Chlorophyta: Tetrasporales, Chlorococcales, Chlorosarcinales). - 352 pp., St. Petersburg.

AnKerT, H. (1917): Schnecken im Winter. - NDMG 49: $140-142$.

Bischoff, H. \& Bold, H.C. (1963): Some soil algae from Enchanted Rock and related algal species. Phycological Studies IV. - Univ. Texas Publ. 6318: 1-95.

CARTER, J.R. (1971): Diatoms from the Devil's Hole Cave, Fife, Scotland. - Nova Hedwigia 21: 657-681.

Dayner, D.M. \& Johansen, J.R. (1991): Observations on the algal flora of Seneca Cavern, Seneca County, Ohio. - Ohio J. Sci. 91: 118-121.

EtrL, H. (1978): Xantophyceae 1. Teil. - In: Etru, H., Gerloff, J. \& Heynig, H. (eds): Süßwasserflora von Mitteleuropa, Vol. 3. - 530 pp., Gustav Fischer Verlag, Jena.

EtTL, H. (1983): Chlorophyta I (Phytomonadina). - In: Ettl, H., Gerloff, J., Heynig, H. \& Mollenhauer, D. (eds): Süßwasserflora von Mitteleuropa, Vol. 9. - 807 pp., Gustav Fischer Verlag, Jena.

Ettl, H. \& GÄrtner, G. (1988): Chlorophyta II (Tetrasporales, Chlorococcales, Gloeodendrales). - In: Ettl, H., Gerloff, J. \& Heynig, H. (eds): Süßwasserflora von Mitteleuropa, Vol. 10. - 436 pp., Gustav Fischer Verlag, Jena.

Ettr, H. \& Gärtner, G. (1995): Syllabus der Boden-, Luft- und Flechtenalgen. - 721 pp., Gustav Fischer, Jena.

Garbacki, N., Ector, L., Kostikov, I. \& Hoffman, L. (1999): Contribution à l'étude de la flore des grottes de Belgique. - Belg. J. Bot. 132: 43-76.

Geitler, L. (1932): Cyanophyceae. - In: Rabenhorst's Kryptogamenflora 14/2. - 1196 pp., Akad. Verlagsges, Leipzig.

Guiry, M.D. \& GuiRY, G.M. 2008. AlgaeBase. Worldwide electronic publication, National University of Ireland, Galway; http://www.algaebase.org; searched on 06 March 2008.

HINDÁK, F. (1996): Klúč na určovanie nerozkonárených vláknitých zelených rias (Ulotrichineae, Ulotrichales, Chlorophyceae). - Bull. Slov. bot. spol., Suppl. 1:1-77.

Houk, V. \& KLEE, R. (2007): Atlas of freshwater centric diatoms with a brief key and descriptions, Part II. Melosiraceae and Aulacoseiraceae (Supplement to Part I). - Fottea 7: 1-255.

Huss, V.A.R., Frank, C., Hartmann, E.C., Hirmer, M., Kloboucek, A., Seidel, B.M., Wenzeler, P. \& Kessler, E. (1999): Biochemical taxonomy and molecular phylogeny of the genus Chlorella sensu lato (Chlorophyta). - J. Phycol. 35: 587-598.
KomáreK, J. \& Anagnostidis, K. (2005): Cyanoprokaryota 2 (Oscillatoriales). - In: BÜDEL, B., Krienitz, L., GÄrtner, G. \& Schagerl, M. (eds): Süßwasserflora von Mitteleuropa, Vol. 19/2. 759 pp., Elsevier/Spektrum, Heidelberg.

KomÁrek, J. \& FotT, B. (1983): Chlorococcales. - In: Huber-Pestalozzi, G. (ed.): Das Phytoplankton des Süßwassers, Vol. 7. - 1043 pp., Schweizerbart, Stuttgart.

KoršiKov, A.A. (1953): Pidklas Protokokovi (Protococcineae). - In: Roll, J.V. (ed.): Viznacnik prisnovodnich vodorostej Ukrainskoj RSR, Vol. 5. - 439 pp., Vidav. akad. Nauk Ukrainskoj RSR.

Kostikov, I., Darienko, T., Lukešová, A. \& Hoffmann, L. (2002): Revision of the classification system of Radiococcaceae Fott ex Komárek (except the subfamily Dictyochlorelloideae) (Chlorophyta). - Algological Studies 104: 23-58.

Krammer, K. (2000): The genus Pinnularia. - In: Lange-Bertalot, H. (ed.): Diatoms of Europe, Vol. 1. 703 pp., A.R.G. Gantner Verlag K.G.

Krammer, K. \& LANGe-Bertalot, H. (1986): Bacillariophyceae 1. Teil. - In: EtTL, H., Gerloff, J., Heynig, H. \& Mollenhauer, D. (eds): Süßwasserflora von Mitteleuropa, Vol. 2/1. -875 pp., Gustav Fischer Verlag, Jena.

Krammer, K. \& Lange-Bertalot, H. (1988): Bacillariophyceae 2. Teil. - In: EtTL, H., Gerloff, J., Heynig, H. \& Mollenhauer, D. (eds): Süßwasserflora von Mitteleuropa, Vol. 2/2. - 596 pp., Gustav Fischer Verlag, Jena.

Krammer, K. \& Lange-Bertalot, H. (1991a): Bacillariophyceae 3. Teil. - In: Ettl, H., Gerloff, J., Heynig, H. \& Mollenhauer, D. (eds): Süßwasserflora von Mitteleuropa, Vol. 2/3. 576 pp., Gustav Fischer Verlag, Stuttgart.

Krammer， K. \& Lange-Bertalot, H. (1991b): Bacillariophyceae 4. Teil. - In: EtTL, H., GÄRTNER, G., Gerloff, J., Heynig, H. \& Mollenhauer, D. (eds): Süßwasserflora von Mitteleuropa, Vol. 2/4. - 436 pp., Gustav Fischer Verlag, Stuttgart.

KrIEGER W. (1937): Die Desmidiaceen Europas mit Berücksichtigung der aussereuropäischen Arten. Rabenhorst's Kryptogamen-Flora von Deutschland, Österreich und der Schweiz, 13, Abt. 1, Teil 1. - 712 pp., Akad. Verlagsges., Leipzig.

Krienitz, L., Hegewald, E.H., Hepperle, D., Huss, V.A.R., Rohr, T. \& Wolf, M. (2004): Phylogenetic relationship of Chlorella and Parachlorella gen. nov. (Chlorophyta, Trebouxiophyceae). - Phycologia 43: 529-542.

KuBÁt, K. (1971): Ledové jámy a exhalace v Českém středohoří II. - Vlastivědný sborník Litoměřicko 8: 67-89.

KUČERA, Z. (1999): Zeměpisné exkurze v západní části 
Severočeského regionu. - Gymnázium Bílina: $8-16$.

Lange-Bertalot, H. (2001): Navicula sensu stricto, 10 genera separated from Navicula sensu lato, Frustulia. - In: Lange-Bertalot, H. (ed.): Diatoms of Europe 2. - 526 pp., A.R.G. Gantner Verlag K.G.

Lauriol, B., Prévost, C. \& Lacelle, D. (2006): The distribution of diatom flora in ice caves of the northern Yukon Territory, Canada: relationship to air circulation and freezing. - Int. J. Spel. 35: 83-92.

LENZENWEGER, R. (1996): Desmidiaceenflora von Österreich, Teil 1. - Bibliotheca Phycologica 101: 1-161.

LOKHORST, G.M.(1996): Comparative taxonomic studies on the genus Klebsormidium (Charophyceae) in Europe. - Crypt. Stud. 5: 1-132.

LožEK, V. (1954): Malakozoologický výzkum reservace Boreč v Českém středohoří. - Ochr. Přír. 9: 9394.

McFarland, M.L., Haby, V.A., Redmon, L.A. \& Bade, D.H. (2001): Managing soil acidity, SCS-200105. -6 pp., Texas A\&M University Agriculture Program FORAGES.

Migula, W. (1907): Algen. - In: Kryptogamen-Flora von Deutschland, Deutsch-Österreich und der Schweiz 2, Teil 1.

NĚMcovÁ, L. (2001): Mechorosty a jejich společenstva na sutích v Českém Středohoří a sousedních územích. - Ph.D. Thesis, Charles Univerity in Prague, Czech Republic.

Pilous, Z. (1959): Mechorosty Státní prrírodní rezervace Borečský vrch v Českém Středohoří. - Ochr. Přír. 14: 97-99.

PoulíčKovÁ, A. \& HaŠler, P. (2007): Aerophytic diatoms from caves in central Moravia (Czech Republic). - Preslia 79: 185-204.

Prescott, G.W., Croasdale, H.T. \& Vinyard W.C. (1972): A synopsis of North American desmids. Part 1, Mesotaeniaceae: Saccodermae. In: Rogerson, C.T. (ed.): North American Flora, II/6. - 84 pp., The New York Botanical Garden.

Printz, H. (1964): Die Chaetophoralen der Binnengewässer. - Hydrobiologia 24: 1-376.

Pujmanová, L. (1990): Mechorosty Borče v Českém středohoří. - Severočes. Přír. 24: 91-96.

RŮŽıČKA, J. (1964): Desmidiaceen der feuchten Felsen in der Hohen Tatra. - Fragm. Flor. et Geobot. 10: 103-119.

RŮŽıČKA, J. (1981): Die Desmidiaceen Mitteleuropas 1. - 736 pp., E. Schweizerbartsche Verlagsbuchhandlung. Stuttgart.

RŮŽıčKA, V. (2003): Beetle communities (Insecta: Coleoptera) of rock debris on the Boreč hill (Czech Republic, České středohoří mountains). - Acta Soc. Zool. Boh. 63: 315-330.
Selvi, B. \& Altuner, Z. (2007): Algae of Ballica Cave (Tokat-Turkey). - IJNES 1: 99-103.

Sluiman, H.J., Guihal, C. \& Mudimu, O. (2008) Assessing phylogenetic affinities and species delimitations in Klebsormidiales (Streptophyta): Nuclear-encoded rDNA phylogenies and its secondary structure models in Klebsormidium, Hormidiella, and Entransia. - J. Phycol. 44: 183-195.

Starmach, K. (1972): Chlorophyta III. Zelenice nitkowate. - In: STARMACH K. (ed.): Flora słodkowodna Polski 10. - 750 pp., PWN, Warszawa \& Krakow.

St. Clair L.L. \& Rushforth S.R. (1976): The diatom flora of Timpanogos Cave National Monument, Utah. - Amer. J. Bot. 63: 49-59.

ŠKALOUD, P. (2008): Images from locality Boreč Hill; http://botany.natur.cuni.cz/skaloud/Borec.htm.

ŠŤASTNÝ, J. (2008): Desmids from ephemeral pools and aerophytic habitats from the Czech Republic. Biologia 63: 884-890.

Tamm, C.O. \& Hallbäcken, L. (1986): Changes in soil ph over a 50-year period under different forest canopies in SW Sweden. - Water Air Soil Pollut. 31: 337-341.

Ter BraAk, C.J.F. \& Šmilauer, P. (1998): CANOCO Reference Manual and User's Guide to Canoco for Windows. - 353pp., Microcomputer Power, Ithaca, USA.

TER BraAk, C.J.F. \& Šmilauer, P. (2002): CANOCO reference manual CanoDraw for Windows user's guide: software for canonical community ordination (version 4.5). - 500 pp., Microcomputer Power. Ithaca, NY, US.

Williamson, D.B. (2000): Some desmid floras of wet rock surfaces. - Algological Studies 97: 11-27.

Wolf, M., Hepperle, D. \& Krienitz, L. (2003): On the phylogeny of Radiococcus, Planktosphaeria and Schizochlamydella (Radiococcaceae, Chlorophyta). - Biologia 58: 759-765.

(C) Czech Phycological Society

Received April 12, 2008

Accepted September 20, 2008 